L'article de Laurence Monnais $(\rightarrow)$ présente une intéressante approche historique des relations entre médecine $(\rightarrow)$ Voir $\mathrm{m} / \mathrm{s}$ février 2017, $33(2): 183-7$ « officielle » et médecines «alternatives » (entités rassemblant de multiples déclinaisons souvent fort éloignées les unes des autres) au cours des XIXe et $x x^{e}$ siècles, et montre que leurs frontières ont souvent été floues et ont évolué au cours du temps. II montre aussi comment le conflit entre ces deux médecines a parfois été instrumentalisé dans une perspective coloniale en déniant toute validité aux savoirs traditionnels « indigènes », et comment certaines pratiques ont pu passer d'un statut à l'autre, comme en témoigne le sort de l'acupuncture, passant du statut de «dispositif thérapeutique parmi d'autres » au XVIII ${ }^{e}$ siècle au rang de thérapeutique à proscrire au siècle suivant, et désormais utilisée pour ses capacités analgésiques et anesthésiques, malgré de nombreuses controverses concernant ses indications.

Malheureusement cet article souffre d'un positionnement très ambigu vis-à-vis de la distinction entre la «médecine fondée sur les preuves » et des approches dont l'efficacité n'a pas été démontrée, et dont les bases théoriques sont souvent contraires aux connaissances les mieux établies (comme l'homéopathie ${ }^{1}$ ). Ces différentes «médecines», qualifiées d'alternatives, parallèles, non-orthodoxes ou complémentaires, outre le fait qu'elles représentent un mélange d'approches très différentes ${ }^{2}$, peu discutées dans l'article ${ }^{3}$, semblent souvent être considérées par l'auteure comme des doctrines ayant a priori la même valeur que la médecine « officielle», sous couvert de «pluralisme médical». L'analyse qui s'ensuit relate les rapports difficiles entre ces différentes pratiques médicales dont la

Bertrand Jordan est l'auteur des «Chroniques Génomiques》 dans médecine/ sciences depuis 1988.

${ }^{1}$ Le seul essai randomisé en double aveugle visant à évaluer le bénéfice clinique de I'utilisation de deux composés homéopathiques (Opium et Raphanus) choisis par les homéopathes en accord avec l'Inserm, sur le rétablissement du transit intestinal après une opération intra-abdominale n'a montré aucune différence significative entre les quatre groupes dans lesquels 600 patients de 12 hôpitaux avaient été répartis [opium + raphanus, opium + placebo, deux placebos, rien (groupe témoin)]. Les résultats de cet essai ont été publiés dans le journal Lancet [Mayaux et al., Lancet, 1988, 1 (8584): 528-9].

${ }^{2}$ Voir le dossier Grand Angle du magazine de l'Inserm Science \& Santé, 2014, 20 : $22-33$

${ }^{3}$ Certaines de ces pratiques médicales peuvent trouver des fondements scientifiques si elles sont explorées avec nos outils de recherche actuels (acupuncture, molécules isolées de la flore ou du règne animal), alors que d'autres ont des bases théoriques - quand elles en ont - contraires à nos connaissance scientifiques.

\section{Relativisme, ambiguités et vérités scientifiques non alternatives}

Bertrand Jordan

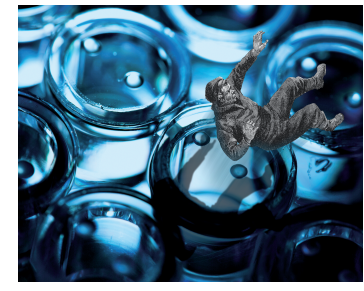

UMR 7268 ADÉS, Aix-Marseille, Université/EFS/CNRS,

Espace éthique méditerranéen, hôpital d'adultes la Timone, 264, rue Saint-Pierre, 13385 Marseille Cedex 05, France ; CoReBio PACA, case 901 , parc scientifique de Luminy, 13288 Marseille Cedex 09, France. bertrand.jordan@univ-amu.fr brjordan@orange.fr

valeur serait équivalente, dans une vision empreinte d'un grand relativisme et dans laquelle la médecine officielle a le mauvais rôle: elle «dénigre », «réprime», use d'《étiquettes diffamatoires », développe une «rhétorique de l'approximation», «sanctionne »le mesmérisme (encore heureux...), exclut... Vision surprenante où est oublié le lent développement de la médecine moderne face à un corpus de rites mêlant religion, pouvoir et thérapies sans preuves, non sans difficultés, ostracisme et répression vis-à-vis de ses artisans, pères d'une médecine enfin efficace ${ }^{4}$. On note aussi, au fil des lignes, une insistance sur les effets secondaires des médicaments (officiels) alors que la toxicité des remèdes naturels (notamment à base de plantes) n'est jamais rappelée, l'évocation de persécutions envers les médecins opposés à la vaccination ${ }^{5}$, l'affirmation que l'accès aux essais cliniques pour les médicaments non «conventionnels » est « obstrué » (par qui ?), suivie quelques lignes plus loin d'un énoncé inverse «il existe dorénavant des médecines alternatives fondées sur les preuves », pour lequel aucune référence n'est fournie.

La grande absente de ce texte est la révolution scientifique de la médecine, qui a débuté vers le milieu du XIXe siècle et qui a transformé l'«art » impuissant des médecins de Molière (traduisant l'ignorance scientifique que cet « art » recouvrait) en une pratique efficace autorisant, avec d'autres facteurs, une augmentation

${ }^{4}$ L'Inquisition fera lourdement payer les malheureux qui s'aventureront à pratiquer des autopsies et dissections sur des cadavres humains, ce qui ralentira considérablement nos connaissances de l'anatomie, ou ceux qui chercheront à comprendre les mécanismes de la circulation sanguine comme Michel Servet, découvreur de la circulation pulmonaire et brûlé vif à Genève en l'an 1553.

${ }^{5}$ Alors que l'éditorial du même numéro de la revue insiste sur l'importance d'améliorer la couverture vaccinale en France... et que l'un des défis actuels est de remobiliser le corps médical pour ce grave problème de santé publique qu'est la diminution des couvertures vaccinales dans les populations. 
continue de l'espérance de vie humaine, avec un recul massif - voire une disparition - de maladies infectieuses comme la diphtérie, la variole et la poliomyélite grâce à la vaccination, avec des avancées majeures dans des domaines comme les maladies cardio-vasculaires. médecine/sciences, notre revue, présente les avancées de cette révolution toujours en marche, mettant en perspective les questions éthiques, sociologiques, et cliniques qu'elles soulèvent, sans sectarisme mais en gardant l'exigence de la rationalité et de preuves expérimentales indiscutables. Cette exigence m'a semblée peu présente dans l'article de Laurence Monnais, et il m'a paru nécessaire de manifester mon désaccord avec sa vision par trop relativiste des rapports entre la médecine fondée sur les preuves et les médecines « alternatives ». $\diamond$

Relativism, ambiguities and non-alternative scientific facts

B ertrand Jordan a participé à l'extraordinaire aventure de la biologie moléculaire, encore balbutiante dans les années 1960, mais qui a complètement révolutionné les sciences du vivant au cours des décennies suivantes.

L'ADN, quasiment inaccessible au début de cette période, intervient maintenant dans d'innombrables recherches, de l'écologie à l'anthropologie, sans oublier bien sûr la médecine dont il fait désormais partie intégrante. Nous pouvons aujourd'hui lire intégralement
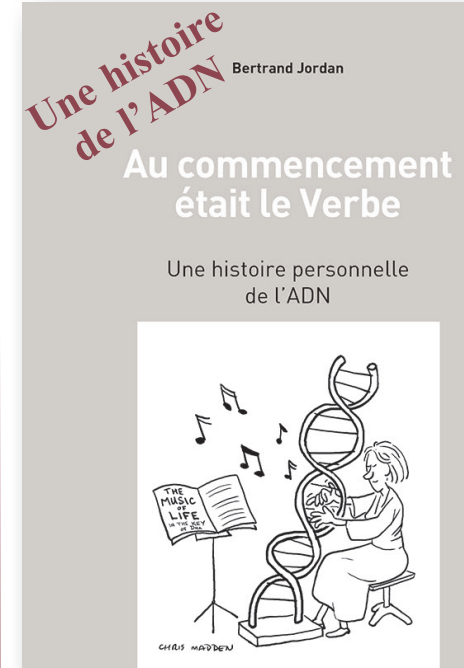

SBN : 978-2-7598-1710-8
110 pages
I'ADN d'une personne en quelques heures, et en tirer de précieuses informations pour la prévention et le traitement de nombreuses maladies - et nous n'en sommes qu'au début de cette nouvelle médecine!

Acteur mais aussi témoin de ces avancées, Bertrand Jordan fait ici un récit très personnel et sans langue de bois de sa vie avec l'ADN. Ce livre ne prétend pas être une histoire complète de la biologie moléculaire, mais il illustre son développement, révélant parfois le «dessous des cartes » grâce aux expériences vécues par son auteur.

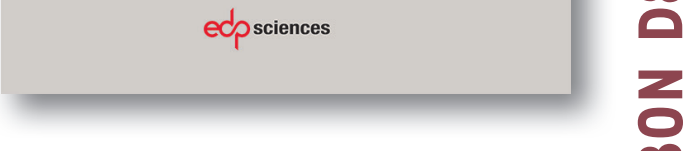

$\delta<$

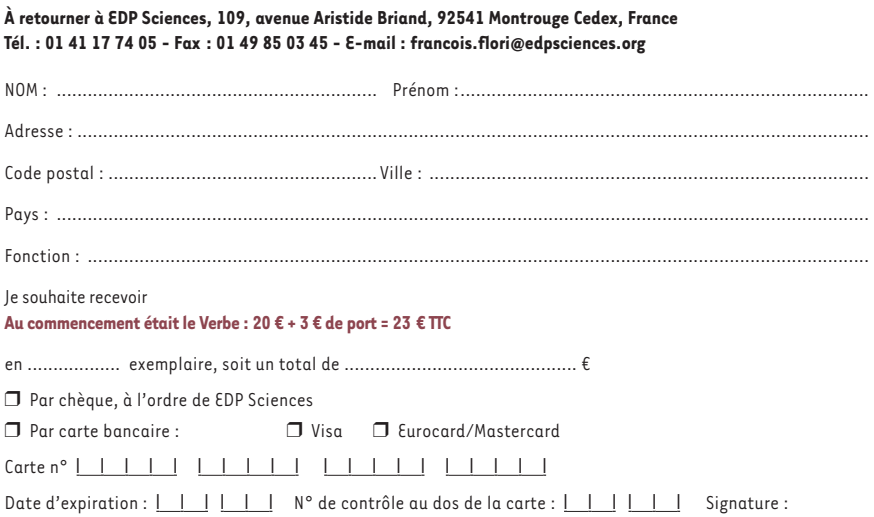

Date d'expiration: ! ! ! ! ! ! $\mathrm{N}^{\circ}$ de contrôle au dos de la carte: ! ! ! ! ! ! Signature

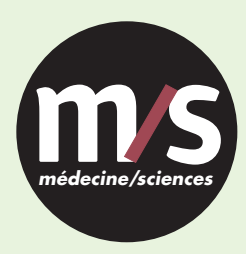

Tarifs d'abonnement $m / s-2017$

Abonnez-vous

à médecine/sciences
> Grâce à $m / s$, vivez en direct les progrès des sciences biologiques et médicales

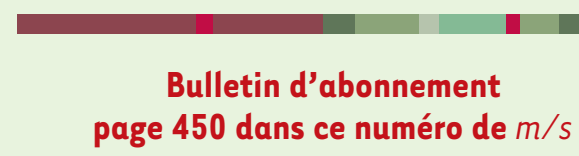

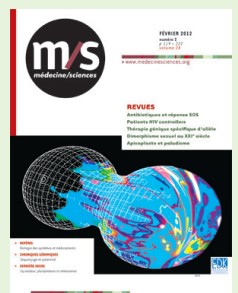

